\title{
Incidental and non-incidental thyroid microcarcinoma
}

\author{
KRZYSZTOF KALISZEWSKI $^{1 *}$, BEATA WOJTCZAK ${ }^{1 *}$, MARTA STRUTYŃSKA-KARPIŃSKA $^{2 *}$, \\ TADEUSZ ŁUKIEŃCZUK ${ }^{1 *}$, ZDZISŁAW FORKASIEWICZ $^{1 *}$ and PAWEŁ DOMOSŁAWSKI ${ }^{1 *}$ \\ ${ }^{1} 1$ st Department and Clinic of General, Gastroenterological and Endocrine Surgery; \\ ${ }^{2}$ Department of Gastrointestinal and General Surgery, Wroclaw Medical University, 50-369 Wroclaw, Poland
}

Received February 15, 2016; Accepted April 13, 2016

DOI: $10.3892 / \mathrm{ol} .2016 .4640$

\begin{abstract}
There is no clear therapeutic approach for thyroid microcarcinoma (TMC). This may be as a consequence of recent observations that have reported biologically different types of TMC, which should be treated differently. The objective of the present study was to compare incidental TMC (ITMC) and non-incidental TMC (NITMC) in order to assess the differences in the incidence, diagnostic results, clinicopathological characteristics and surgical treatment. The study consisted of a retrospective chart review of 3,218 patients consecutively admitted and surgically treated in a single institution due to thyroid pathology. A total of 246 (7.64\%) patients presented with a thyroid malignancy, and 97 (39.43\%) of these individuals were diagnosed with TMC; 37 (38.14\%) patients exhibited ITMC and $60(61.86 \%)$ exhibited NITMC. All $37(100.00 \%)$ patients with ITMC exhibited a papillary type of cancer. In the NITMC group, $1(1.67 \%)$ patient presented with follicular microcarcinoma, $1(1.67 \%)$ individual with papillary- and follicular-type microcarcinoma, 1 (1.67\%) individual with medullary microcarcinoma, and the remaining $57(95.00 \%)$ patients presented with papillary microcarcinoma. The number of younger patients ( $<45$ years old) was higher in the NITMC group, but this difference was not significant $(\mathrm{P}=0.205)$. In all patients with ITMC, ultrasound-guided fine-needle aspiration biopsy did not reveal malignant processes. In the NITMC group, the number of larger tumors $(>5 \mathrm{~mm})$ was significantly higher compared with that in the ITMC group $(\mathrm{P}<0.001)$. ITMC was significantly associated with multinodular goiter (MNG) $(\mathrm{P}<0.001)$. Amongst the NITMC group, $18.33 \%$ of patients presented with cervical lymph node involvement $(\mathrm{P}<0.001)$. Overall, the incidence of TMC is high, and consists of ITMC and NITMC, although the prevalence of NITMC is higher than that of ITMC. The majority of ITMCs and NITMCs are composed of a papillary
\end{abstract}

Correspondence to: Dr Krzysztof Kaliszewski, 1st Department and Clinic of General, Gastroenterological and Endocrine Surgery, Wroclaw Medical University, 66 Sklodowskiej-Curie Street, 50-369 Wroclaw, Poland

E-mail: krzysztofkali@wp.pl

*Contributed equally

Key words: thyroid, cancer, incidental, microcarcinoma type of cancer. The majority of ITMCs have dimensions $\leq 5 \mathrm{~mm}$ in diameter and are significantly associated with MNG. In comparison with ITMC, NITMC more often presents with aggressive features such as cervical lymph node metastases, so the surgical approach to this tumor should be more radical.

\section{Introduction}

The prevalence of thyroid cancer (TC) is rising (1). In Poland in 2000, the number of new cases of this malignancy amounted to 1,457 individuals, while in 2010, this number was 2,192 individuals (1,808 women and 384 men). TC accounts for 2.6 and $0.5 \%$ of all oncological diseases in women and men, respectively (2). In other countries, its prevalence varies from 2-36\%, which supports the hypothesis that the incidence of TC depends on the geographical region (3). During the last two decades, the morbidity rate of TC in Poland has markedly increased (2).

One specific type of this malignancy is thyroid microcarcinoma (TMC). According to the World Health Organization, TMC is defined as TC measuring $\leq 10 \mathrm{~mm}$ at its greatest dimension (1). The clinical and pathological significance of TMC is widely debated. It is common for TMC to be diagnosed upon histopathological examination after surgery that has been performed for presumed benign thyroid disease (4).

The prevalence of incidental TMC (ITMC) is variable, ranging from 4.6-100\% of TMCs in the general population $(5,6)$. In multinodular goiter (MNG), ITMC has been observed in $2-15.2 \%$ of cases (7). The different results of the prevalence of ITMC are likely observed due to the various geographical regions, but also due to variable use of ultrasonography and ultrasound-guided fine needle aspiration biopsy (UG-FNAB), and the different experience levels of researchers (8). Regardless of diagnostic method, it has been suggested that a constantly increasing incidence of TMC is present in the general population (9). Cappelli et al (10) noted that the prevalence of ITMC in patients who underwent UG-FNAB with negative results for the detection of TC was $1.24 \%$.

A number of studies have shown that an increasing number of patients surgically treated for presurgically diagnosed TC had a tumor size $<5 \mathrm{~mm}(4-7,9)$; this may therefore be the reason for the constant increase in the prevalence of TMC. With regard to this malignancy, another specific type of thyroid tumor has been distinguished; these tumors are not 
evident clinically, are $<1 \mathrm{~cm}$ in diameter and are found after lymph node biopsy or as an incidental finding upon autopsy examinations (11). The tumors are described as occult TMC. The high incidence of TMC found upon autopsy studies (up to $35 \%$ ) may suggest that the majority of these tumors exhibit benign behavior (12).

Generally, it is known that the papillary type of TMC follows a benign clinical course, however, the proper therapeutic management in these tumors is a subject of debate. It has been suggested that there are three biologically different types of papillary TMC (PTMC) and that they should be treated differently (13). The first type consists of an incidentally detected PTMC without any symptoms, and whose clinical course is excellent $(12,13)$. Conservative follow-up is indicated in these carcinomas (13). The second type involves the early stage of the usual low-risk PTMC, and the third type comprises high-risk cancer and is clinically symptomatic $(11,13)$. The treatment of the aforementioned carcinomas varies from a lobectomy to a total thyroidectomy with or without redioiodine therapy (12-14). Küçük et al (12) suggested that the non-incidental papillary type of TMC should be treated as a conventional carcinoma with a total thyroidectomy, followed by radioiodine therapy in certain cases. Therefore, the widely debated clinical scenario concerns the proper surgical treatment of TMC recognized after surgery. When a primary surgical procedure is radical, further management may be required. Similarly, if the initial surgery is non-radical a radicalization may be required later on, which is resection of the remnant thyroid tissue after primary surgery. Occasionally, however, a 'borderline' situation occurs and a dilemma is raised. Therefore, therapeutic protocols and follow-up algorithms require modification (14). The present study aimed to compare ITMC and NITMC in order to assess the differences in the incidence, diagnostic results, clinicopathological characteristics and surgical treatments.

\section{Patients and methods}

Patients and approval. The present study was a retrospective chart review of 3,218 patients consecutively admitted and surgically treated in the 1st Department and Clinic of General, Gastroenterological and Endocrine Surgery (Wroclaw Medical University) between January 1, 2008, and November 30, 2014 , due to the presence of thyroid tumors. For histopathological examination and immunohistochemical analysis (IHC), hematoxylin and eosin stained sections were evaluated by two independent pathologists to confirm the diagnosis of TMC (according to the World Health Organization Classification system) (1) and the AJCC/UICC 2010 staging system (6). For IHC, $4-\mu \mathrm{m}$-thick sections were cut from the specimens. Each slide was immunostained with monoclonal antibodies against cytokeratin-19 (Dako, Glostrup, Denmark). IHC was performed using the EnVision ${ }^{\mathrm{TM}}$ FLEX kit (Dako) and the chromogen 3,3'-diaminobenzidine as a substrate. Finally, all slides were counterstained with Mayer's hematoxylin according to the manufacturer's protocol. All immunostaining was evaluated by two pathologists using a BX-41 light microscope (Olympus Corporation, Tokyo, Japan). The study was approved by the Bioethics Committee of Wroclaw Medical University (Wroclaw, Poland). Demographic data, diagnostic results, clinical and histopathological characteristics, surgical treatment and necessity of reoperation were evaluated. As a main presurgical diagnostic test, all patients in the ITMC and NITMC groups underwent UG-FNAB.

Statistical analysis. The statistical significance of the differences in patient age between the ITMC and NITMC groups was verified with the aid of a t-test, and the statistical significance of the differences in the proportions of studied subclasses between the ITMC and NITMC groups was defined with the aid of the $\chi^{2}$ test or Fisher's exact test (when the subclass size was $<5$ ). The statistical analysis was performed with the use of Statistica 7.1 (Dell Software, Tulsa, OK, USA) and PAST version $1.86 \mathrm{~b}$ software (folk.uio.no/ohammer/past/terms.html).

\section{Results}

Patient cohort and demographic characteristics. A total of $246(7.64 \%)$ patients with thyroid malignancy were evaluated (out of 3,218 patients with benign and malignant thyroid tumors), and 97 (3.01\% of total thyroid pathology; $39.43 \%$ of TC patients) of these were diagnosed with TMC. In this homogenous group were 37 (38.14\%) patients with ITMC and $60(61.86 \%)$ with NITMC. There were $33(89.19 \%)$ females and $4(10.81 \%)$ males in the ITMC group, and $57(95.00 \%)$ females and $3(5.00 \%)$ males in the NITMC group. The mean ages ( \pm standard deviation) of the patients in the ITMC and NITMC groups were $51.45( \pm 13.026)$ and $46.93( \pm 15.317)$, respectively; this difference was not statistically significant.

Pathological characteristics. All 37 (100.00\%) patients in the ITMC group exhibited a papillary type of cancer. In the NITMC group, $1(1.67 \%)$ patient presented with a follicular type of TMC, $1(1.67 \%)$ with a tumor composed of a papillary and follicular type of cancer, and $1(1.61 \%)$ with a medullary type of TMC. The remaining $57(95.00 \%)$ patients presented with a papillary type of TMC.

Clinical characteristics. In all 60 (100.00\%) patients with NITMC, the diagnosis of suspected malignancy was estimated on the basis of UG-FNAB. In all 37 (100.00\%) individuals with ITMC, UG-FNAB procedures did not reveal malignant processes. The number of younger patients ( $<45$ years old) was higher in the NITMC group than in the ITMC group, but this difference was not statistically significant $(\mathrm{P}=0.20)$. In the NITMC group, the proportion of patients with larger tumors ( $>5 \mathrm{~mm}$ ) was significantly higher compared with that in the ITMC group $(\mathrm{P}<0.001)$. The tumors size of the patients with ITMC and NITMC estimated upon histopathological examination (pT) are presented in Table I. ITMC was significantly associated with MNG $(\mathrm{P}<0.001)$. Amongst the NITMC group, $11(18.33 \%)$ patients presented with cervical lymph node involvement $(\mathrm{pN} 1 \mathrm{a})(\mathrm{P}<0.001)$

All analyzed data, together with detailed results of the statistical analysis, are presented in Table II.

\section{Discussion}

The prevalence of ITMC is rising and ranges from 4.6-100\% of TMCs in the general population $(5,6)$. The American 
Table I. Size of the tumors (ITMC vs. NITMC) estimated upon histopathological examination (pT).

Tumor size, $\mathrm{mm}$

\begin{tabular}{|c|c|c|c|c|c|c|c|c|c|c|c|}
\hline \multirow[b]{2}{*}{ Type of cancer } & \multicolumn{5}{|c|}{$\leq 5 \mathrm{~mm}$} & \multicolumn{5}{|c|}{$>5 \mathrm{~mm}$} & \multirow[b]{2}{*}{ Total } \\
\hline & $\leq 1$ & $1.1-2$ & $2.1-3$ & $3.1-4$ & $4.1-5$ & $5.1-6$ & $6.1-7$ & $7.1-8$ & $8.1-9$ & $9.1-10$ & \\
\hline \multicolumn{12}{|l|}{ ITMC, $\mathrm{n}$} \\
\hline Papillary & 2 & 3 & 3 & 6 & 8 & 4 & 1 & 4 & 2 & 4 & 37 \\
\hline \multicolumn{12}{|l|}{ NITMC, $\mathrm{n}$} \\
\hline Papillary & 0 & 0 & 1 & 3 & 5 & 8 & 6 & 8 & 9 & 17 & 57 \\
\hline Follicular & 0 & 0 & 0 & 0 & 0 & 0 & 0 & 0 & 0 & 1 & 1 \\
\hline Papillary/follicular & 0 & 0 & 0 & 0 & 0 & 0 & 0 & 0 & 0 & 1 & 1 \\
\hline Medullary & 0 & 0 & 0 & 0 & 0 & 1 & 0 & 0 & 0 & 0 & 1 \\
\hline Total, n (\%) & \multicolumn{5}{|c|}{$31(31.96)$} & \multicolumn{5}{|c|}{$66(68.04)$} & \\
\hline
\end{tabular}

ITMC, incidental thyroid microcarcinoma; NITMC, non-incidental thyroid microcarcinoma.

Thyroid Association (ATA) (15) and the American Association of Clinical Endocrinologists suggest that small nodules ( $\leq 10 \mathrm{~mm}$ in diameter) should be biopsied only when clinical and ultrasound characteristics such as microcalcifications, irregular margins, a history of neck and head irradiation, or a positive history for family cancer are present (15-17). This recommendation should decrease the number of ITMC cases and increase the diagnosis of NITMC cases. The 2015 ATA TC management guidelines accept an active surveillance management approach as an alternative to immediate UG-FNAB and surgery in such nodules and also in the cytologically confirmed very low risk papillary type of TMC (18). However, the ATA guidelines provide no specific recommendation regarding the selection of the patients who can be qualified to an active surveillance management approach. One previous study suggested that solitary nodules of $<10 \mathrm{~mm}$ in diameter should be treated as potentially malignant tumors (19). The study also suggested that nodules $\leq 2 \mathrm{~mm}$ in diameter may be biopsied too, as often sufficient cytological material can be obtained. In the present study, in the NITMC group, the smallest nodule in which UG-FNAB was successfully performed, sufficient diagnostic material was obtained and a malignant process was revealed, measured $3 \mathrm{~mm}$ in diameter (Table I). Generally, the median size of TMC in each patient varies from 4.1-8.0 $\mathrm{mm}$ in diameter, but cases of microcarcinoma that have a size $<1 \mathrm{~mm}$ have also been described (20). In the present study, in the ITMC group, 3 cases with a tumor size of $1.5 \mathrm{~mm}$ and 2 patients with a TMC measuring $1 \mathrm{~mm}$ in diameter were evaluated. All these tumors were of papillary type. Noguchi et al (19) presented an autopsy study of the thyroid gland of patients from different geographical areas, and revealed the higher prevalence of TMCs 1-3 mm in diameter versus those 4-9 $\mathrm{mm}$ in diameter, accounting for 50.4 and $27.3 \%$ of cases, respectively. The study suggested that this could be the result of the 'arrest of the growth' of the papillary type of TMC. Notably, Fukunaga and Yatani (20) described the sclerosing process within PTMCs. This study suggested that the process was a defensive mechanism of tumor growth. This may be the reason that this variant of PTMC is rarely observed in the group of larger PTMCs. In the present study, it was estimated that smaller tumors $(\leq 5 \mathrm{~mm})$ were significantly associated with ITMC. In the ITMC group of patients, tumors $\leq 5 \mathrm{~mm}$ in size were diagnosed in $22(59.46 \%)$ individuals. In the NITMC group of patients, tumors $\leq 5 \mathrm{~mm}$ in diameter were only diagnosed in $9(15.00 \%)$ individuals $(\mathrm{P}<0.001)$.

The mean age at diagnosis of patients with ITMC and NITMC has been widely reported (21). The younger age of $<45$ years is often associated with NITMC. In the present study, in the group of patients with NITMC, there was a higher number of patients aged $<45$ years compared with the number in the ITMC group, but this difference was not statistically significant (36.67 vs. $24.32 \%$, respectively; $\mathrm{P}=0.205$ ).

The majority of TMC is PTMC, which accounts for $65-99 \%$ of all cases. The follicular type has been found in $0.3-23.6 \%$ of all cases (21). In the present study, PTMC accounted for $96.91 \%$ of all TMCs. In the group of ITMC all $37(100 \%)$ tumors were of the papillary type. Non-papillary TMC types were observed in all NITMC cases; this group consisted of 1 $(1.67 \%)$ patient with the follicular type, 1 (1.67\%) individual with a tumor composed of the follicular and papillary type, and $1(1.67 \%)$ patient with the medullary type of TMC. This last tumor measured $6 \mathrm{~mm}$ in diameter and was recorded in a 49-year-old female with TMC diagnosed prior to surgery (NITMC group).

In two different studies performed at different times, Pellegriti et al (11) and Lupoli et al (22) each described familial cases of TMC. Each study noted that PTMC may be observed in members of the same family, reporting 7 and 10 familial cases, respectively. Lupoli et al (22) suggested that familial cases of TMC are more aggressive than non-hereditary cases, but this was not confirmed by other studies. Certain studies confirmed that the outcomes of the familial type of papillary TC, compared with PTMC, differed from that of sporadic tumors, so more invasive surgery was recommended in this type of malignancy $(23,24)$. With regard to familial and sporadic TMC, the complete opposite was observed; familial PTMC was revealed to be less aggressive, and less invasive surgical treatment was indicated for consideration. In the present study, 
Table II. Demographic and clinicopathological characteristics of patients with ITMC $(n=37)$ vs. NITMC $(n=60)$.

\begin{tabular}{|c|c|c|c|}
\hline Parameter & ITMC & NITMC & Statistics and significance \\
\hline Age in years, mean $\pm \mathrm{SD}$ & $51.46 \pm 13.03$ & $46.93 \pm 15.32$ & t-test: $\mathrm{t}=-1.49 ; \mathrm{df}=59 ; \mathrm{P}=0.138$ \\
\hline Younger/older, n (\%) & & & $\chi^{2}$ test: $\chi^{2}=1.60 ; \mathrm{df}=1 ; \mathrm{P}=0.205$ \\
\hline$<45$ years & $9(24.32)$ & $22(36.67)$ & \\
\hline$\geq 45$ years & $28(75.68)$ & $38(63.33)$ & \\
\hline Gender, n (\%) & & & Fisher's exact test: $\mathrm{P}=0.422$ \\
\hline Female & 33 (89.19) & $57(95.00)$ & \\
\hline Male & $4(10.81)$ & $3(5.00)$ & \\
\hline \multicolumn{4}{|l|}{ UG-FNAB, n (\%) } \\
\hline Suspicion & $0(0.00)$ & $60(100.00)$ & \\
\hline No suspicion & $37(100.00)$ & $0(0.00)$ & \\
\hline \multicolumn{3}{|l|}{ Tumor size, n (\%) } & $\chi^{2}$ test: $\chi^{2}=20.80 ; \mathrm{df}=1 ; \mathrm{P}<0.001$ \\
\hline$\leq 5 \mathrm{~mm}$ & $22(59.46)$ & $9(15.00)$ & \\
\hline$>5 \mathrm{~mm}$ & $15(40.54)$ & $51(85.00)$ & \\
\hline \multicolumn{3}{|l|}{ Type of lesion, n (\%) } & Fisher's exact test: $\mathrm{P}<0.001$ \\
\hline MNG & $36(97.30)$ & $35(58.33)$ & \\
\hline Solitary tumor & $1(2.70)$ & $25(41.67)$ & \\
\hline \multicolumn{3}{|l|}{ Histopathology, n (\%) } & Fisher's exact test: $\mathrm{P}=0.704$ \\
\hline Papillary & $37(100.00)$ & $57(95.00)$ & \\
\hline Papillary/follicular & $0(0.00)$ & $1(1.67)$ & \\
\hline Follicular & $0(0.00)$ & $1(1.67)$ & \\
\hline Medullary & $0(0.00)$ & $1(1.67)$ & \\
\hline \multicolumn{3}{|l|}{ Multifocality, n (\%) } & Fisher's exact test: $\mathrm{P}=0.122$ \\
\hline Solitary & 35 (94.59) & $49(81.67)$ & \\
\hline Multifocal & $2(5.41)$ & $11(18.33)$ & \\
\hline \multicolumn{4}{|l|}{ Familial cases, n (\%) } \\
\hline Yes & $0(0.00)$ & $0(0.00)$ & \\
\hline No & $37(100.00)$ & $60(100.00)$ & \\
\hline \multicolumn{3}{|l|}{ Type of surgery, n (\%) } & $\chi^{2}$ test: $\chi^{2}=40.66 ; \mathrm{df}=1 ; \mathrm{P}<0.001$ \\
\hline Radical & $9(24.32)$ & $53(88.33)$ & \\
\hline Non-radical & $28(75.68)$ & $7(11.67)$ & \\
\hline \multicolumn{3}{|l|}{ Reoperation required, n (\%) } & Fisher's exact test: $\mathrm{P}=0.080$ \\
\hline No & $31(83.78)$ & $57(95.00)$ & \\
\hline Yes & $6(16.22)$ & $3(5.00)$ & \\
\hline \multicolumn{3}{|l|}{ Cervical lymph node involvement, n (\%) } & Fisher's exact test: $\mathrm{P}<0.001$ \\
\hline pN0 & $2(5.41)$ & $49(81.67)$ & (pN0 vs. pN1a-pNx) \\
\hline pN1a & $0(0.00)$ & $11(18.33)$ & \\
\hline $\mathrm{pN} 1 \mathrm{~b}$ & $1(2.70)$ & $0(0.00)$ & \\
\hline $\mathrm{pNx}$ & $34(91.89)$ & $0(0.00)$ & \\
\hline Total & $37(38.14)$ & $60(61.86)$ & \\
\hline
\end{tabular}

Data is presented as the mean \pm standard deviation. ITMC, incidental thyroid microcarcinoma; NITMC, non-incidental thyroid microcarcinoma; UG-FNAB, ultrasound-guided fine-needle aspiration biopsy; MNG, multinodular goiter; df, degrees of freedom.

in the two groups of patients (ITMC and NITMC), no familial cases were observed. All TMCs were sporadic types.

Sakorafas et al (25) suggested that amongst all the clinical and histopathological characteristics of ITMC and NITMC, for further evaluation, the most important characteristics are multifocality and bilaterality. These histopathological features are observed in 7.1-56.8 and 2.9-48\% of ITMC and NITMC cases, respectively. In the present study, multifocality was observed in $5.4 \%$ of ITMC cases and in $18.3 \%$ of NITMC cases. This difference was statistically significant $(\mathrm{P}<0.001)$. The next significant characteristic is lymph nodes metastases, which have been observed in 2-62.1 and 0-64\% of ITMC and 
NITMC cases, respectively (26). In the present study, cervical lymph node involvement was observed in 2.70 and $18.33 \%$ of ITMC and NITMC cases, respectively. Ito et al (27) stated that $\sim 40 \%$ of patients with the papillary type of NITMC possessed lymph node metastases, as diagnosed after surgery, even though all patients had been classified as $\mathrm{cN} 0$ prior to surgery. Distant metastases are observed extremely rarely and account for $\sim 0.37 \%$ of cases (8). In the present patient groups, this characteristic was not observed. Certain studies reported that cervical and distant metastases occurred more often in patients with NITMC than in patients with ITMC (28). Ito et al confirmed that the incidence of lymph node metastases is higher in patients with NITMC compared with in those with ITMC (27), which was also confirmed in the present study. Another study added that patients with larger ITMC and NITMC tumors had a higher probability of the presence of lymph node metastases at the time of diagnosis than patients with smaller tumors (29). Fu et al (30) suggested that the tumor size had a prognostic value in the papillary type of ITMC and NITMC, and that a larger size was associated with a higher risk of central neck lymph node metastases. In the present patients with NITMC, of the $11(18.33 \%)$ individuals with cervical lymph node involvement, $6(10 \%)$ patients presented with tumors $>5 \mathrm{~mm}$ in diameter. All of these patients had metastases to the central lymph node compartment. Kim et al (31) revealed that the factors predictive of central lymph node metastases in patients with this type of malignancy differed by age. The study suggested that the size of such a tumor was an independent predictor of subclinical central lymph node involvement only in patients aged $\geq 45$ years. In the present study, in the group of patients with central lymph node metastases, 6 (54.55\%) patients were $<45$ years and all of these patients were in the NITMC group. In total, 5 (8.33\%) patients in the NITMC group and $1(2.70 \%)$ individual in the ITMC group were $\geq 45$ years old. Zhang et al (32) revealed that male gender and tumor size ( $\geq 6 \mathrm{~mm}$ ) were risk factors of central compartment lymph node metastases, and therefore recommended a prophylactic central lymph node dissection in patients with this type of TMC and the aforementioned risk factors. Another study (33) suggested that a central compartment lymph node dissection should be considered in all patients with NITMC, as even in patients without these risk factors, the incidence of central lymph node metastases is higher than expected. The study also stated that the complication rate of radical surgery with central lymph node dissection should be at an acceptable level.

One previous study indicated that the higher prevalence of lymph node metastases is connected with the follicular variant of PTMC (28). It was suggested that the coexistence of Hashimoto's thyroiditis is protective for lymph node metastases. In the present study, $1(1.67 \%)$ patient with the follicular type of NITMC and 1 (1.67\%) patient with NITMC composed of the papillary and follicular types were observed. These 2 patients presented with cervical lymph node involvement. No inflammatory processed were observed within the thyroid tissues in either group.

A meta-analysis by Mehanna et al (34) revealed the existence of a minimum of two different entities with regard to the papillary types of ITMC and NITMC. It was suggested that incidental tumors had distinct clinical characteristics and a much lower recurrence rate than non-incidental lesions.
Furthermore, it was suggested that management protocols for these two different groups of cancers should be reconsidered. ITMCs have significantly fewer aggressive characteristics than conventional TC. NITMC, which presents with aggressive features like conventional TC, should be treated in a similar manner and a routine total thyroidectomy with central lymph node compartment dissection should be performed (35). Similar observations were noted in a study by Vasileiadis et al (36), which recorded 339 individuals with the papillary type of TMC, accounting for $12 \%$ of all patients who underwent surgery for thyroid disease. It was noted that bilaterality, autoimmune thyroid disease, a tumor size of $>5 \mathrm{~mm}$ in diameter, multifocality and lymph node metastasis were significantly associated with non-incidental tumors. It was concluded that the majority of NITMCs (papillary type) present at the time of diagnosis possessed one or more risk factors, including multifocality, bilaterality or greater tumor size; therefore, a total thyroidectomy is recommended, followed by adequate exploration of the central lymph node compartment to provide a safe therapeutic approach. Another study suggested that tumor size, a non-incidental diagnosis and neck node metastasis at the time of diagnosis were independent risk factors for aggressive NITMC (6). A further study revealed that NITMC tumors are associated with a higher cancer stage at the time of diagnosis, and therefore more invasive behavior and a higher tendency for recurrence. As a result, aggressive surgical treatment was recommended in cases of such tumors (37). In the present study, $60(100.00 \%)$ patients with NITMC qualified to undergo radical surgery with central lymph node dissection. Of these, $53(88.33 \%)$ patients underwent a radical procedure and $7(11.67 \%)$ of underwent a non-radical procedure. In the group of patients with ITMC, only $9(24.32 \%)$ patients underwent a total thyroidectomy. In total, 3 (5.00\%) patients with NITMC and $6(16.22 \%)$ patients with ITMC required radicalization, which is resection of the remnant thyroid tissue after primary surgery.

In the literature, ITMC is significantly associated with MNG, while NITMC is often found within normal or Hashimoto's thyroiditis $(38,39)$. In the present study, ITMCs were significantly associated with $\mathrm{MNG}(\mathrm{P}<0.001)$ and only $1(2.70 \%)$ patient in this group underwent surgery due to a solitary tumor. No coexisting autoimmunological process was observed within the thyroid gland in either the ITMC or NITMC group.

Notable observations concerning the surgical treatment of ITMC and NITMC were presented by Gschwandtner et al (40). To evaluate a less-radical approach, the study revealed that a non-incidental finding, nodal metastases at presentation, a young age, aggregate tumor size and subcapsular tumor localization were risk factors for lymph node metastases, and therefore did not recommend surgical treatment. Multifocality, gender, maximum tumor size and the extent of surgery were not noted to be relevant factors. In the majority of PTMC cases, even with multifocality, a limited surgical approach without completion thyroidectomy, lymphadenectomy or radioiodine treatment was recommended as sufficient management. However, it was stated that in cases of intra- or pre-operative clinically suspected lymph node metastases, or in cases with post-operatively diagnosed risk factors, a standard radical procedure should be performed (40). In the present study, 
in the group of patients with NITMC, 57 (95.00\%) radical surgeries were performed without the necessity of a second surgery. In the group of patients with ITMC, radical procedures were performed in $31(83.78 \%)$ cases, and $6(16.22 \%)$ individuals required radicalization, due to coexisting risk factors, including multifocality or bilaterality. Only $3(5.00 \%)$ patients in the NITMC group required a reoperation.

The present study concluded that: i) The incidence of TMC is high and consists of ITMC and NITMC cases; ii) the prevalence of NITMC is higher than that of ITMC; iii) the majority of ITMCs and NITMCs are of papillary type; iv) the majority of ITMCs have a diameter $\leq 5 \mathrm{~mm}$; v) ITMC is significantly associated with MNG; and vi) NITMC compared with ITMC often presents with aggressive features such as cervical lymph node metastases, therefore a surgical approach in this tumor should be more radical.

\section{References}

1. Elliott MS, Gao K, Gupta R, Chua EL, Gargya A and Clark J: Management of incidental and non-incidental papillary thyroid microcarcinoma. J Laryngol Otol 127 (Suppl 2): S17-S23, 2013.

2. Didkowska J: Epidemiology of malignancy in Poland and in the World. In: Compendium of Surgical Oncology. Jeziorski A and Rutkowski P (eds). Via Med, Gdańsk, pp3-18, 2014.

3. Solares CA, Penalonzo MA, Xu M and Orellana E: Occult papillary thyroid carcinoma in postmortem species: Prevalence at autopsy. Am J Otolaryngol 26: 87-90, 2005.

4. Yassa L, Cibas ES, Benson CB, Frates MC, Doubilet PM, Gawande AA, Moore FD Jr, Kim BW, Nosé V, Marqusee E, et al: Long-term assessment of a multidisciplinary approach to thyroid nodule diagnostic evaluation. Cancer 111: 508-516, 2007.

5. Lim DJ, Baek KH, Lee YS, Park WC, Kim MK, Kang MI, Jeon HM, Lee JM, Yun-Cha B, Lee KW, et al: Clinical, histopathological, and molecular characteristics of papillary thyroid microcarcinoma. Thyroid 17: 883-888, 2007.

6. Lombardi CP, Bellantone R, De Crea C, Paladino NC, Fadda G, Salvatori M and Raffaelli M: Papillary thyroid microcarcinoma: Extrathyroidal extension, lymph node metastases, and risk factors for recurrence in a high prevalence of goiter area. World J Surg 34: 1214-1221, 2010.

7. Abdulmughni YA, Al-Hureibi MA, Al-Hureibi KA, Ghafoor MA, Al-Wadan AH and Al-Hureibi YA: Thyroid cancer in Yemen. Saudi Med J 25: 55-59, 2004.

8. Roti E, degli Uberti EC, Bondanelli M and Braverman LE: Thyroid papillary microcarcinoma: A descriptive and meta-analysis study. Eur J Endocrinol 159: 659-673, 2008.

9. Davies L and Welch HG: Increasing incidence of thyroid cancer in the United States, 1973-2002. JAMA 295: 2164-2167, 2006.

10. Cappelli C, Castellano M, Braga M, Gandossi E, Pirola I, De Martino E, Agosti B and Rosei EA: Aggressiveness and outcome of papillary thyroid carcinoma (PTC) versus microcarcinoma (PMC): A mono-institutional experience. J Surg Oncol 95: 555-560, 2007.

11. Pellegriti G, Scollo C, Lumera G, Regalbuto C, Vigneri R and Belfiore A: Clinical behavior and outcome of papillary thyroid cancers smaller than $1.5 \mathrm{~cm}$ in diameter: Study of 299 cases. J Clin Endocrinol Metabol 89: 3713-3720, 2004.

12. Küçük NO, Tari P, Tokmak E and Aras G: Treatment for microcarcinoma of the thyroid-clinical experience. Clin Nucl Med 32: 279-281, 2007

13. Sugitani I, Toda K, Yamada K, Yamamoto N, Ikenaga M and Fujimoto Y: Three distinctly different kinds of papillary thyroid microcarcinoma should be recognized: Our treatment strategies and outcomes. World J Surg 34: 1222-1231, 2010.

14. Feng $\mathrm{J}$ and Wang J: Diagnosis and treatment of thyroid microcarcinoma. Lin Chung Er Bi Yan Hou Tou Jing Wai Ke Za Zhi 28 1911-1917, 2014 (In Chinese).

15. Haugen BR, Alexander EK, Bible KC, et al: 2015 American Thyroid Association Management Guidelines for Adult Patients with Thyroid Nodules and Differentiated Thyroid Cancer: The American Thyroid Association Guidelines Task Force on Thyroid Nodules and Differentiated Thyroid Cancer. Thyroid 26: $1-133,2016$.
16. Carlini M, Giovannini C, Castaldi F, Mercadante E, Dell'Avanzato R, Zazza S, Nania A, Santeusanio G, Passeri M and Di Perna P: High risk for microcarcinoma in thyroid benign diseases. Incidence in a one year period of total thyroidectomies. J Exp Clin Cancer Res 24: 231-236, 2005.

17. Gharib H, Papini E, Paschke R, Duick DS, Valcavi R, Hegedüs L and Vitti P; AACE/AME/ETA Task Force on Thyroid Nodules: American Association of Clinical Endocrinologists, Associazione Medici Endocrinologi, and European Thyroid Association medical guidelines for clinical practice for the diagnosis and management of thyroid nodules: Executive summary of recommendations. J Endocrinol Invest 33: 51-56, 2010.

18. Brito JP, Ito Y, Miyauchi A and Tuttle RM: A clinical framework to facilitate risk stratification when considering an active surveillance alternative to immediate biopsy and surgery in papillary microcarcinoma. Thyroid 26: 144-149, 2016.

19. Noguchi S, Yamashita H, Murakami N, Nakayama I, Toda M and Kawamoto H: Small carcinomas of the thyroid. A longterm follow-up of 867 patients. Arch Surg 131: 187-191, 1996.

20. Fukunaga FH and Yatani R: Geographic pathology of occult thyroid carcinomas. Cancer 36: 1095-1099, 1975.

21. Pisello F, Geraci G, Sciumè C, Li Volsi F and Modica G: Total thyroidectomy of choice in papillary microcarcinoma. G Chir 28: $13-19,2007$

22. Lupoli G, Vitale G, Caraglia M, Fittipaldi MR, Abbruzzese A, Tagliaferri P and Bianco AR: Familial papillary thyroid microcarcinoma: A new clinical entity. Lancet 353: 637-639, 1999.

23. Sung TY, Lee YM, Yoon JH, Chung KW and Hong SJ: Surgical management of familial papillary thyroid microcarcinoma: A single institution study of 94 cases. World J Surg 39: 1930-1935, 2015.

24. Yang GC, LiVolsi VA and Baloch ZW: Thyroid microcarcinoma: Fine needle aspiration diagnosis and histologic follow-up. Int J Surg Pathol 10: 133-139, 2002.

25. Sakorafas GH, Stafyla V, Kolettis T, Tolumis G, Kassaras G and Peros G: Microscopic papillary thyroid cancer as an incidental finding in patients treated surgically for presumably benign thyroid disease. J Postgrad Med 53: 23-26, 2007.

26. Dietlein M, Luyken WA, Schicha $\mathrm{H}$ and Larena-Avellaneda A: Incidental multifocal papillary microcarcinomas of the thyroid: Is subtotal thyroidectomy combined with radioiodine ablation enough? Nucl Med Commun 26: 3-8, 2005.

27. Ito Y, Higashiyama T, Takamura Y, Miya A, Kobayashi K, Matsuzuka F, Kuma K and Miyauchi A: Risk factors for recurrence to the lymph node in papillary thyroid carcinoma patients without preoperatively detectable lateral node metastasis: Validity of prophylactic modified radical neck dissection. World J Surg 31: 2085-2091, 2007.

28. Roti E, Rossi R, Trasforini G, Bertelli F, Ambrosio MR, Busutti L, Pearce EN, Braverman LE and Degli Uberti EC: Clinical and histological characteristics of papillary thyroid microcarcinoma: Results of a retrospective study in 243 patients. J Clin Endocrinol Metab 91: 2171-2178, 2006

29. Machens A, Holzhausen HJ and Dralle H: The prognostic value of primary tumor size in papillary and follicular thyroid carcinoma. Cancer 103: 2269-2273, 2005.

30. Fu X, Lou S, Shi H, Liu Q, Chen Z and Zhou Y: Clinicopathologic analysis of 254 cases of papillary thyroid microcarcinoma. Zhonghua Bing Li Xue Za Zhi 44: 258-261, 2015 (In Chinese).

31. Kim JY, Jung EJ, Park T, Jeong SH, Jeong CY, Ju YT, Lee YJ, Hong SC, Choi SK and Ha WS: Impact of tumor size on subclinical central lymph node metastasis in papillary thyroid microcarcinoma depends on age. World J Surg Oncol 13: 88 , 2015.

32. Zhang LY, Liu ZW, Liu YW, Gao WS and Zheng CJ: Risk factors for nodal metastasis in $\mathrm{cNO}$ papillary thyroid microcarcinoma. Asian Pac J Cancer Prev 16: 3361-3363, 2015.

33. Chang YW, Kim HS, Kim HY, Lee JB, Bae JW and Son GS: Should central lymph node dissection be considered for all papillary thyroid microcarcinoma? Asian J Surg: April 22, 2015 (Epub ahead of print).

34. Mehanna H, Al-Maqbili T, Carter B, Martin E, Campain N, Watkinson J, McCabe C, Boelaert $\mathrm{K}$ and Franklyn JA: Differences in the recurrence and mortality outcomes rates of incidental and nonincidental papillary thyroid microcarcinoma: A systematic review and meta-analysis of 21329 person-years of follow-up. J Clin Endocrinol Metab 99: 2834-2843, 2014.

35. Karatzas T, Vasileiadis I, Kapetanakis S, et al: Risk factors contributing to the difference in prognosis for papillary versus micropapillary thyroid carcinoma. Am J Surg 206: 586-593, 2013. 
36. Vasileiadis I, Karatzas T, Vasileiadis D, Kapetanakis S Charitoudis G, Karakostas E and Kouraklis G: Clinical and pathological characteristics of incidental and nonincidental papillary thyroid microcarcinoma in 339 patients. Head Neck 36: 564-570, 2014.

37. Arora N, Turbendian HK, Kato MA, Moo TA, Zarnegar R and Fahey TJ III: Papillary thyroid carcinoma and microcarcinoma: Is there a need to distinguish the two? Thyroid 19: 473-477,2009.

38. Pisanu A, Reccia I, Nardello O and Uccheddu A: Risk factors for nodal metastasis and recurrence among patients with papillary thyroid microcarcinoma: Differences in clinical relevance between nonincidental and incidental tumors. World J Surg 33: 460-468, 2009.
39. Slijepcevic N, Zivaljevic V, Marinkovic J, Sipetic S, Diklic A and Paunovic I: Retrospective evaluation of the incidental finding of 403 papillary thyroid microcarcinomas in 2466 patients undergoing thyroid surgery for presumed benign thyroid disease. BMC Cancer 15: 330, 2015.

40. Gschwandtner E, Klatte T, Swietek N, Bures C, Kober F, Ott J, Schultheis A, Neuhold N and Hermann M: Increase of papillary thyroid microcarcinoma and a plea for restrictive treatment: A retrospective study of 1,391 prospective documented patients. Surgery 159: 503-511, 2016. 